\title{
Stable Cold-Field Emission from Tungsten (310) in an SEM Gun with an Additional Non-Evaporative Getter Pump
}

\author{
K. Kasuya, ${ }^{*}$ M. Kimura,** S. Katagiri,* and T. Ohshima* \\ * Central Research Laboratory, Hitachi, Ltd., 1-280 Higashi-Koigakubo Kokubunji, Tokyo, 185- \\ 8601, Japan \\ ** Hitachi High-Technologies Corp., 882 Ichige, Hitachinaka, Ibaraki, 312-8504, Japan
}

Owing to their superior properties of high brightness and low energy spread, cold-field-emission (CFE) guns are widely used in high-resolution SEMs, TEMs, and STEMs. In spite of these advantages, a problem associated with CFE has been instability of its emission current. That is, current decay and current noise. This instability originates from adsorption of residual gases from ambient vacuum. Accumulating gas adsorption on the emitter surface increases the surface work function, thereby resulting in current decay. The current noise comes from migration or desorption of adsorbed gases [1]. A solution to this problem is to decrease vacuum pressure. Field emissions under extreme-high vacuum were reported previously $[2,3]$. In this study, stable field emission from a tungsten (310) emitter in an SEM gun evacuated by an additional non-evaporative getter (NEG) pump was demonstrated.

A conventional CFE gun for SEM (Hitachi S-7000), equipped with an ion pump and NEG pump [4], was used. Minimum pressure of the gun was in the order of $10^{-10} \mathrm{~Pa}$. Figure 1 shows total current and probe current of emission at $2 \times 10^{-9} \mathrm{~Pa}$. The probe current was the confined current of $1 \times 10^{-4} \mathrm{sr}$ at the center of the total current. The pressure decrease by the NEG pump prolonged current decay. In the beginning of probe emission right after flashing, there was a current period with small decay and low noise, which continued for several hours until the decay began (the "decreasing region" in FIG.1). This period is hereafter called "stable-bright-region". Electrons in the stable-bright-region are assumed to be emitted from a clean surface or a surface with few adsorbed molecules. The amount of current increases compared to that in the conventionally used "stable region", which follows the decreasing region. Energy spread is possibly lowered in the stable-bright-region. Duration of the stable-bright-region depends on vacuum pressure; that is, decreasing pressure extends the period of stable-bright-region.

Figure 2 shows probe current and corresponding time dependence of the emission pattern of the tungsten (310) measured by another experimental setup. Emission intensity from the (310) remained almost constant from the start of emission in accordance with stable-bright-region. In contrast, the intensity of the (100) and (211) weakened with time. This anisotropy of current decay with crystal faces explains the difference between the damping behavior of the total and probe currents.

Most of the gases absorbed in the period of stable-bright-region are assumed to be hydrogen, since hydrogen is the main component under extreme- or ultra-high vacuum. Hydrogen desorbs from tungsten at $400^{\circ} \mathrm{C}$ [5], so the stable-bright-region can be continued by intermittent "mild flashing" at temperature lower than the conventional $2000^{\circ} \mathrm{C}$. Figure 3 shows probe current under intermittent mild flashing. It is clear that stable-bright-region is successively continued at high current and low noise for 12 hours and more. It is concluded from this result that use of the stable-bright-region improves stability of CFE and attains superior electron-microscope performance and usability [6]. 


\section{References}

[1] H. Todokoro et al., Jpn. J. Appl. Phys. 21 (1982) 1513.

[2] B. Cho et al., Appl. Phys. Lett. 91 (2007) 012105.

[3] T. Tomita et al., Microsc. Microanal. 15 (Suppl. 2) (2009) 1084.

[4] S. Katagiri et al., Microelectronics Engineering 83 (2006) 1058.

[5] P. W. Tamm et al., J. Chem. Phys. 52 (1970) 1150.

[6] Acknowledgement: The authors thank S. Kokubo and Dr. B. Cho of Hitachi High-Technologies Corp. for the helpful discussions of research and development.

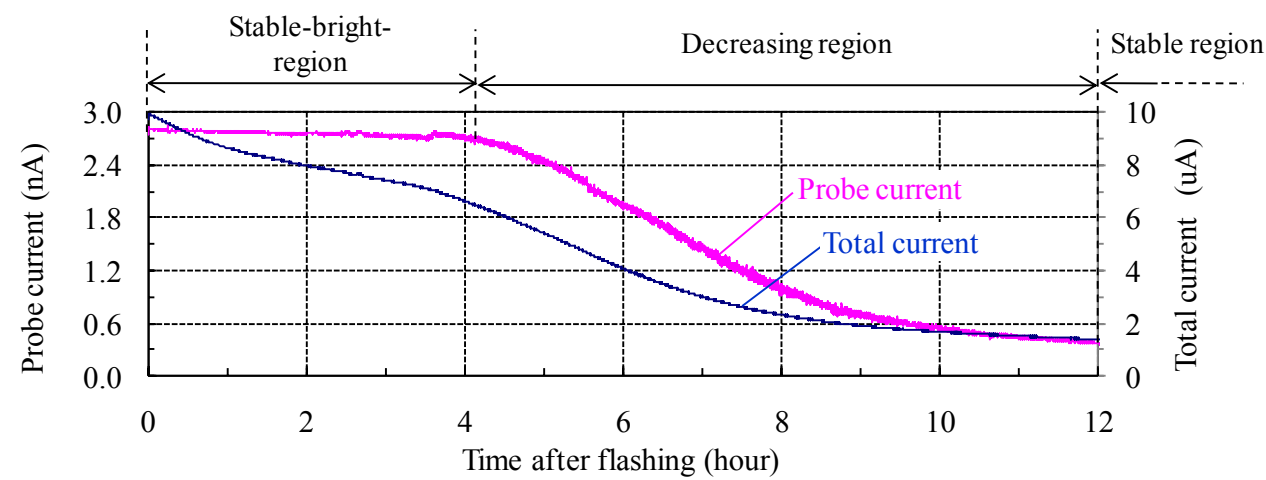

FIG. 1. Total current and probe current of tungsten (310) field emission at $2 \times 10^{-9} \mathrm{~Pa}$.

(a)

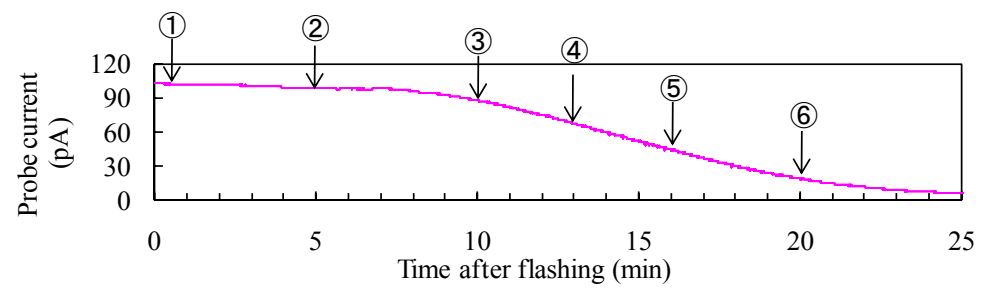

(b)

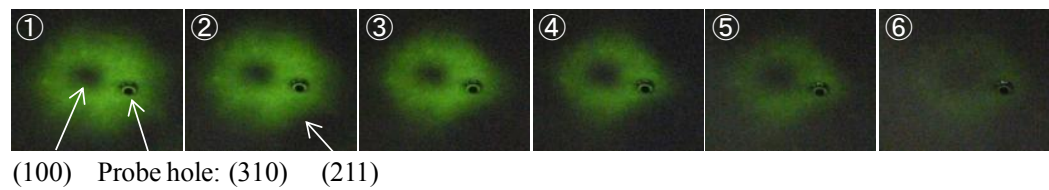

FIG. 2. (a) Time dependence of probe current and (b) corresponding emission pattern of tungsten (310) emitter. Numbers indicated in (a) correspond to each picture in (b). The probe current was corrected through a probe hole on a fluorescent screen.

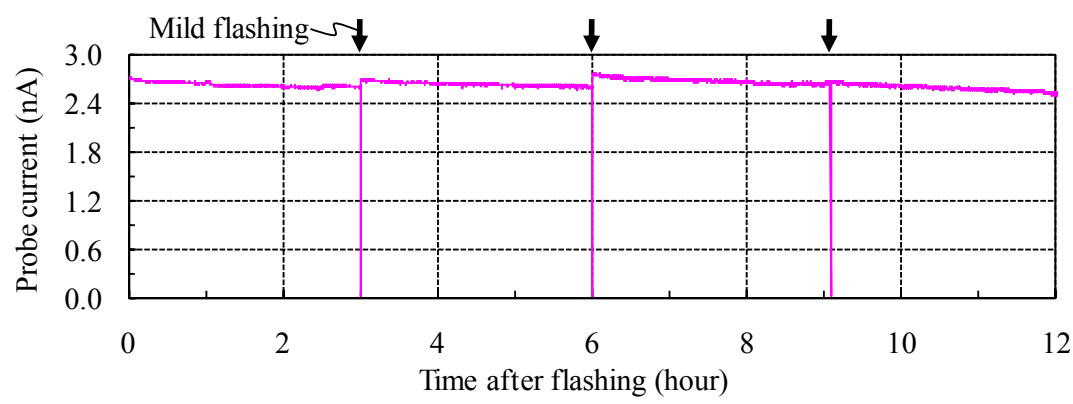

FIG. 3. Probe current continued through the stable-bright-region by intermittent mild flashing at three-hour intervals. Arrows above the graph indicate the time when mild flashing was done. 